\title{
Preoperative assessment of the older surgical patient: honing in on geriatric syndromes
}

This article was published in the following Dove Press journal:

Clinical Interventions in Aging

16 December 2014

Number of times this article has been viewed

\author{
Sunghye Kim' \\ Amber K Brooks ${ }^{2}$ \\ Leanne Groban² \\ 'Department of Hospital Medicine, \\ 2Department of Anesthesiology, \\ Wake Forest School of Medicine, \\ Winston-Salem, NC, USA
}

\begin{abstract}
Nearly 50\% of Americans will have an operation after the age of 65 years. Traditional preoperative anesthesia consultations capture only some of the information needed to identify older patients (defined as $\geq 65$ years of age) undergoing elective surgery who are at increased risk for postoperative complications, prolonged hospital stays, and delayed or hampered functional recovery. As a catalyst to this review, we compared traditional risk scores (eg, cardiac-focused) to geriatric-specific risk measures from two older female patients seen in our preoperative clinic who were scheduled for elective, robotic-assisted hysterectomies. Despite having a lower cardiac risk index and Charlson comorbidity score, the younger of the two patients presented with more subtle negative geriatric-specific risk predictors - including intermediate or pre-frail status, borderline malnutrition, and reduced functional/mobility - which may have contributed to her 1-day-longer length of stay and need for readmission. Adequate screening of physiologic and cognitive reserves in older patients scheduled for surgery could identify at-risk, vulnerable elders and enable proactive perioperative management strategies (eg, strength, balance, and mobility prehabilitation) to reduce adverse postoperative outcomes and readmissions. Here, we describe our initial two cases and review the stress response to surgery and the impact of advanced age on this response as well as preoperative geriatric assessments, including frailty, nutrition, physical function, cognition, and mood state tests that may better predict postoperative outcomes in older adults. A brief overview of the literature on anesthetic techniques that may influence geriatric-related syndromes is also presented.
\end{abstract}

Keywords: frailty, mobility-disability, postoperative delirium, preoperative evaluation, stress response

\section{Case I: ML}

An 81-year-old woman presented to the preoperative assessment clinic (PAC) prior to an elective robotic total hysterectomy for biopsy-proven adenocarcinoma of the endometrium, FIGO grade 2. She had a medical history of rheumatic heart disease, with a previous mitral valve replacement with a tissue valve, atrial fibrillation with chronic anticoagulation and pacemaker placement, and coronary artery disease. She denied any cardiac symptoms except for mild shortness of breath on exertion. The patient was taking metoprolol, valsartan, aspirin, simvastatin, and warfarin. Her blood pressure was $150 / 81 \mathrm{mmHg}$, heart rate was 78 beats/min, and oxygen saturation was $98 \%$ on room air. Her heart beat was irregularly irregular without murmurs. Her lung sounds were clear. There was no pitting edema. Per the Revised Cardiac Risk Index (RCRI) for preoperative risk, the patient had one risk factor (coronary artery disease) with an expected risk of having a major postsurgical adverse cardiac event of $0.9 \%-1.1 \%$. Per the American College of Cardiology/American Heart Association (ACC/AHA) guidelines on perioperative cardiac evaluation, she underwent robotic total laparoscopic hysterectomy, bilateral salpingo-oophorectomy, and pelvic lymph
Correspondence: Leanne Groban Department of Anesthesiology, Wake Forest School of Medicine, Medical Center Boulevard, Winston-Salem, NC 27I57-1009, USA

$\mathrm{Tel}+\mathrm{I} 3367164498$

Fax +13367168190

Email lgroban@wakehealth.edu 
node dissection, with continuation of metoprolol for heart rate control. Perioperatively, she was also bridged with low molecular weight heparin and unfractionated heparin. Her postoperative course was uneventful, and she was discharged on postoperative day (POD) 1 to home with home health services. She returned to the gynecology clinic 1 month later for surgical follow-up and was found to be doing well without any overt complications.

\section{Case 2: KM}

A 72-year-old woman presented to the PAC prior to her scheduled robotic total hysterectomy for complex atypical hyperplasia of the endometrium. Her medical history included benign essential hypertension, schizophrenia, gastroesophageal reflux disease, and irritable bowel syndrome. She was taking hydrochlorothiazide, labetalol, olanzapine, lovastatin, trazodone, lansoprazole, amlodipine, potassium chloride, and vitamin E. She denied any cardiovascular (CV) symptoms. Her schizophrenia was well controlled on antipsychotic medications and, although she has received assistance from a social worker, she was independent on all her activities and instrumental activities of daily living (ADLs and IADLs). In the PAC, her blood pressure was 136/64 $\mathrm{mmHg}$, heart rate was 74 beats/min, and oxygen saturation was $98 \%$ on room air. Her heart beat was regular without murmurs. Her lung sounds were clear. There was no pitting edema. Per the RCRI, the patient had no major CV risk factors and the expected risk of having a major adverse postoperative cardiac event was $0.4 \%-0.5 \%$. Per the ACC/ AHA guidelines on perioperative cardiac evaluation, it was advised that she proceed with the surgery without further work-up. She underwent a robotic total hysterectomy, bilateral salpingo-oophorectomy, ureteral stent placement, and pelvic washing. The pathology later was reported as stage I adenocarcinoma grade 2 . Her immediate postoperative course was uncomplicated, and she was discharged, accompanied by her son, to her home on POD 2, without home health services. Unfortunately, on POD 32, she was admitted to the hospital after sustaining a fall in her apartment and was found to have an open tarsometatarsal fracture dislocation of her left foot. She underwent a closed reduction and debridement, and was discharged to a skilled nursing facility.

\section{Discussion}

We describe the cases of two older female patients who underwent uncomplicated gynecologic-oncologic hysterectomies via robotic assistance. While both patients were considered to have an American Society of Anesthesiologists (ASA) physical status of 3 , per traditional cardiac risk assessment measures, the 81-year-old patient (ML) was deemed to be at a higher risk than the 72-year-old patient (KM). ML had other comorbidities that increased her Charlson comorbidity score to 6 compared to a score of 4 for KM. Even so, the length of stay (LOS) was 1 day longer for the younger patient and her postsurgical course was questionable. Both patients were enrolled in our prospective cohort study of older noncardiac surgical patients, allowing us to determine whether other preoperative measures, such as frailty scores, nutritional status, and mobility/disability scores, might better predict these patients' postoperative outcomes (Table 1). ML's metabolic equivalent task (MET) score was 4.2, with an estimated weekly energy expenditure from activities (eg, doing light work around the house like dusting or washing dishes) of $996 \mathrm{kcal}$. Her body mass index (BMI) was $26 \mathrm{~kg} / \mathrm{m}^{2}$, and her mini-nutritional assessment-short form (MNA-sf) score was 14 . Her high-sensitivity $C$ reactive protein (CRP) was $0.53 \mathrm{mg} / \mathrm{L}$ and her 25 hydroxy-vitamin D was $25.2 \mathrm{ng} / \mathrm{dL}$.

Patient KM's MET was 1.3, with estimated weekly energy consumption from activities (eg, ADL) of $409 \mathrm{kcal}$. Her BMI was $35 \mathrm{~kg} / \mathrm{m}^{2}$ and her MNA was 14 . Her frailty

Table I Geriatric-specific preoperative evaluation tests

\begin{tabular}{lll}
\hline & ML & KM \\
\hline Metabolic equivalent task score (METS) & 4.2 & 1.3 \\
Mobility (MAT-sf) & 48.05 & 36.9 \\
Nutrition (MNA-sf) & 14 & 9 \\
Frailty score & $\mathrm{I}($ Not frail) & 3 (Intermediately frail) \\
Pain score & 0 & 0 \\
Estimated weekly energy expenditure & $996 \mathrm{kcal}$ & $409 \mathrm{kcal}$ \\
BMI & $26 \mathrm{~kg} / \mathrm{m}^{2}$ & $35 \mathrm{~kg} / \mathrm{m}^{2}$ \\
C-reactive protein (hs-CRP) & $0.53 \mathrm{mg} / \mathrm{L}$ & $57 \mathrm{mg} / \mathrm{L}$ \\
Vitamin D & $25.2 \mathrm{ng} / \mathrm{mL}$ & $7.1 \mathrm{ng} / \mathrm{mL}$ \\
\hline
\end{tabular}

Abbreviations: MNA, mini-nutritional survey scored 0-14 (increasing score $=$ better nutritional status); MAT-sf, mobility assessment tool-short form, scored 30-69 (increasing score = better mobility); Frailty scored $0-5$ (increased score = increased frailty vulnerability); Pain, scored numerically $0-10$ (increasing score = increased pain); BMI, body mass index; hs-CRP, high-sensitivity C-reactive protein $(>1 \mathrm{mg} / \mathrm{L}=$ increased inflammation); Vitamin $\mathrm{D}$, serum 25 -hydroxyvitamin $\mathrm{D}[25(\mathrm{OH}) \mathrm{D}](\leq 20 \mathrm{ng} / \mathrm{mL}=$ deficiency; $2 \mathrm{I}-29$ insufficiency, and $\geq 30 \mathrm{ng} / \mathrm{mL}$ sufficient). 
score was 3 and her MAT-sf score was 37. Her CRP was $57 \mathrm{mg} / \mathrm{L}$ and vitamin D was $7.1 \mathrm{ng} / \mathrm{dL}$. Indeed, her history of schizophrenia and long-term use of atypical antipsychotics might have been the most compelling factor for her increased frailty and reduced mobility. Prolonged intellectual disability (ID) and treatment with antipsychotics have been associated with the development of early frailty, ${ }^{1}$ and this may be due, in part, to the metabolic symptoms, eg increased BMI, higher diastolic blood pressure, and elevated fasting glucose, that often develop following chronic treatment of ID. ${ }^{2,3}$

\section{Case for expanding preoperative assessment for older patients}

Traditional perioperative risk assessment measures may often overlook subtle geriatric-specific syndromes that translate into increased vulnerabilities for older patients. In current medical practice, patients scheduled for elective surgery are evaluated preoperatively to assess the patients' risk of adverse postsurgical outcomes given their underlying medical conditions. Since the first scientific attempt to predict postoperative outcome, ${ }^{4}$ multiple predictive scoring systems have been developed and validated..$^{5-8}$ Most of these preoperative assessment tools rely on existing comorbidities, exercise tolerance, and some lab values to predict postoperative outcomes. Since most of these variables can be obtained through the patient interview and the electronic medical records, these preoperative risk tools are very easy to use and have good accuracy in predicting perioperative complications. However, none of these tools considers physiologic characteristics specific to elderly patients. In addition, the outcomes of interest that they focus on are primarily based on single-organ systems (eg, cardiac, pulmonary). Furthermore, most of the tools currently available are unable to accurately predict outcomes such as LOS, functional recovery, or need for institutionalization; all of these are key outcomes that may help guide decision making processes specifically for older surgical patients, their families, and physicians. ${ }^{9}$

With the aging population, it is expected that Americans aged 65 years and older will grow from $13 \%$ to $>20 \%$ of the total population by year $2030 .{ }^{10}$ More than one-third of all inpatient surgical procedures in the US were performed on patients aged 65 years and older in 2007, and this number is expect to double by $2020 .{ }^{11,12}$ Some of the procedures done on older adults are life-saving measures (eg, cancer resection), while other surgeries are done to improve quality of life (eg, joint replacement). Having a clear understanding of postoperative recovery trajectories and postoperative complication rates is important so that appropriate discussions can take place between patients, their families, and physicians, and realistic treatment goals can be set and achieved. Normal age-related physiologic changes such as impaired left ventricular compliance, stiffening of the systemic vasculature, decreased lung mechanics, and reduced renal function limit the physiologic reserve of older patients and make them more vulnerable to postoperative stress and illness. ${ }^{12}$ As a result, elderly patients have higher postoperative complication rates, ${ }^{12,13}$ and it is known that surgical management is often compromised in elderly patients. ${ }^{14}$ For the remainder of this review, we will describe the stress response to surgery and the impact of advanced age on this response, and discuss various preoperative geriatric assessments that may better predict postoperative outcomes in older adults.

\section{Surgical stress response and aging}

Surgery can have life-altering effects extending beyond that of the original diagnosis for which the surgery was performed. In elderly patients in particular, postsurgical stress, both physical and psychological, can lead to an imbalance in autonomic, endocrine, metabolic, and immune functions. Depending on the patient's preoperative physiologic reserve and comorbid conditions, additional clinical challenges may impose further alterations in the stress response and, thus, the recovery trajectory. ${ }^{15,16}$ Here we describe some agerelated changes in the surgical stress response that lower the homeostatic threshold in older patients, increasing their risk of postoperative adverse events and complicating their return to independence after surgery.

Among the systemic responses to surgery is an activation of the sympathetic autonomic nervous system (SNS). Hypothalamic activation of the SNS by afferent neural input from the injured area leads to increased secretion of epinephrine from the adrenal medulla and release of norepinephrine (NE) from presynaptic nerve endings. ${ }^{17} \mathrm{NE}$ is a neurotransmitter; it spills over into the systemic circulation, further modifying the functions of the liver, adrenal cortex, pancreas, and kidneys. The significance of SNS activation lies in its direct actions on the myocardium and vasculature, which can result in hypertension, tachycardia, and, potentially, dysrhythmias. ${ }^{18}$ Taken together, these CV changes may lead to myocardial ischemia in susceptible patients due to an increase in oxygen demand. Direct activation of cardiac sympathetic nerves may also trigger coronary vasoconstriction in patients with atherosclerotic coronary artery disease. Moreover, circulating catecholamines may contribute to a hypercoagulable state, further burdening the ischemic potential of those more vulnerable patients with preexisting coronary artery disease. In the older 
patient, these responses to surgery also occur in the context of a higher prevalence of $\mathrm{CV}$ and pulmonary diseases, which are known to increase with age. Diminished arterial and left ventricular compliance, ${ }^{19,20}$ impaired vasoconstriction, altered autonomic function and sensitivity to catecholamines, ${ }^{21}$ and decreased baroreflex sensitivity ${ }^{22}$ may all impair the maintenance of CV homeostasis during acute surgical illness in the elderly. In addition to an activated SNS, increases in anterior and posterior pituitary hormone, aldosterone, cortisol, and glucagon secretion also contribute to the surgical stress response. ${ }^{23}$ The hypothalamic-pituitary-adrenal (HPA) axis stimulates secretion of the growth hormone $(\mathrm{GH})$ and the adrenocorticotrophic hormone (ACTH). GH, in turn, stimulates protein synthesis and inhibits protein breakdown, promotes lipolysis, stimulates glycogenolysis in the liver, and has an anti-insulin effect by inhibiting glucose uptake and use by cells. In response to ACTH stimulation, cortisol secreted from the adrenal cortex promotes protein breakdown and lipolysis, which increases the production of gluconeogenic precursors. In contrast to the catabolic hyperglycemic response to surgery, insulin concentrations may fall during surgery, in part due to adrenergic inhibition of pancreatic beta-cell secretion. This is further confounded by transitory "insulin resistance," which occurs during the perioperative period. ${ }^{18}$ Notably, these hormonal responses reflect the degree of surgical stress; hormonal responses are negligible during low-risk procedures and are transient, lasting no longer than 24 hours after elective, uncomplicated surgery. Endocrine responses to elective surgical procedures are intact in older patients in terms of plasma cortisol levels and urinary excretion of adrenaline, noradrenaline, and 17-hydroxycorticosteroids, ${ }^{24}$ and the increases in energy expenditure that occur after such procedures are independent of the patient's age.

Even in low-risk surgeries, many of the age-related changes in metabolic responses to surgical illness can be attributed to alterations in body composition and physical activity characteristic of the elderly. Although weight remains relatively stable, fat mass tends to increase with age while muscle mass tends to decrease. The loss of strength that accompanies immobility, starvation, and acute surgical illness may have marked functional consequences in the postoperative period. The capacity of muscle to function as an energy source in the postoperative period may be limited in the elderly patient, ${ }^{25}$ and muscle strength may become inadequate for respiratory and other vital muscle function. In patients undergoing elective major gastrointestinal surgery, a strong relationship between protein depletion and postoperative pulmonary complications has been reported. ${ }^{26}$
In addition to producing a catabolic state, amplified secretion of catecholamines and cortisol due to surgical stress has both inflammatory and immunosuppressant actions. ${ }^{27,28}$ Cortisol blocks the production of cytokines, prostaglandins, and histamines, and it interferes with the aggregation of neutrophils and macrophages at the site of tissue trauma and decreases phagocytosis. ${ }^{29} \mathrm{Cortisol}$ also promotes helper T-cell-2 (Th2) dominance, or a decrease in Th1/Th2 ratio, which is responsible, in part, for depressed cellular immunity after surgery. ${ }^{27}$

Tissue trauma as a result of surgery also contributes to a profound inflammatory response near the surgical site, elevating cellular mediators, or cytokines, which further exacerbate inflammation and suppress the immune response. The proinflammatory cytokines, tumor necrosis factor- $\alpha$ (TNF- $\alpha$ ) and interleukin-1 (IL-1), are released from activated macrophages and monocytes in damaged tissues and stimulate the production of interleukin-6 (IL-6). ${ }^{30}$ This cytokine is responsible for producing the systemic changes referred to as the acute-phase response, including fever, granulocytosis, and the production of acute-phase liver proteins, eg, CRP, fibrinogen, and $\alpha 2$-macroglobulin. ${ }^{30}$ The release of IL-1, IL-6, TNF- $\alpha$, and acute-phase reactants can activate the HPA axis; taken together, these changes lead to an imbalance in the Th1/Th2 ratio and postsurgical immunosuppression. ${ }^{31}$

Whether a causal relationship exists between postsurgical immunosuppression and an age-related increase in susceptibility to postoperative complications, such as cognitive dysfunction, thromboembolic events, and lung infections, is not clear. While immune function is depressed with aging, ${ }^{32}$ the oldest of the old have been shown to be in a chronic state of inflammation. ${ }^{33,34}$ A 2-4-fold higher level of proinflammatory cytokines, leukocytes, and acute-phase reactants such as CRP have been reported in the elderly. ${ }^{35,36}$ With regard to surgery and anesthesia, older patients undergoing low-risk hernia surgery showed an initial hyperactive response followed by a delayed termination of inflammatory activity, defined by leukocyte and neutrophil numbers and CRP, TNF- $\alpha$, and IL-1 levels, when compared to younger patients undergoing the same surgery.

Taken together, surgical procedures lead to a variety of physiological alterations characterized by changes in hemodynamic, endocrine, and immune function. While the magnitude of the stress response depends on several surgical, anesthetic, and patient-related factors such as severity and duration of the surgical trauma, ${ }^{15,37}$ surgical technique, ${ }^{38,39}$ anesthetic method, ${ }^{23,40,41}$ and the patient's age and comorbid conditions, the presence of subtle geriatric-related 
syndromes, such as frailty, mobility-disability, and malnutrition should also be considered, as they may compromise the older patient's homeostatic compensatory mechanisms necessary for combating periprocedural stress.

\section{Frailty status}

One characteristic of the aging body is a reduction in physiologic reserve, or frailty. Frailty is broadly defined as a state of increased vulnerability resulting from age-associated declines in reserve and function across multiple physiologic systems, such that the ability to cope with everyday or acute stressors is compromised..$^{42}$ Frailty is prevalent among elderly populations and is a recognized risk factor for adverse health outcomes, including mortality, institutionalization, and falls. ${ }^{43-45}$ There are two phenotypes of frailty described in the literature. The physical phenotype of frailty, defined by Fried et al, ${ }^{45}$ is a clinical syndrome in which three or more of the following criteria are present: unintentional weight loss (10 lbs in past year), self-reported exhaustion, weakness (grip strength), slow walking speed, and low physical activity. The multidomain phenotype of frailty considers additional components such as cognitive impairment, mood disorders, sensory impairment, poor social conditions and support, chronic diseases, and disability as part of the frailty syndrome. The multidomain phenotype is usually based on a comprehensive geriatric assessment, such as the Canadian Study of Health and Aging Frailty Index, which consists of 70 items. ${ }^{46,47}$ The Edmonton Frailty Scale (EFS) is another frailty assessment tool with ten domains, namely cognition, general health status (two questionnaires), functional independence, social support, medication use, nutrition, mood, continence, and functional performance, with two of the domains involving performance-based items. ${ }^{48}$ Interestingly, EFS includes social support as one of the domains. ${ }^{48}$ Using EFS as a measure of frailty, Dasgupta et $\mathrm{al}^{49}$ found that, in 125 patients who were undergoing elective noncardiac surgery, increasing frailty was associated with postoperative complications, increased LOS, and inability to be discharged home. On the other hand, Makary et $\mathrm{al}^{50}$ used the Hopkins Frailty Score, a modified fivedomain frailty scale developed by Fried et $\mathrm{al}^{45}$ which comprises weakness, weight loss, exhaustion, physical activity, and walking speed to identify frail (score of 4 or 5) and intermediately frail patients (score of 2 or 3 ). Using this score, they found that out of 594 patients aged 65 years and older having elective noncardiac surgery, $10.4 \%$ were frail and $31.3 \%$ were intermediately frail. Preoperative frailty was associated with an increased risk of surgical complications, extended LOS, and discharge to a skilled or assisted living facility.
When frailty was added to other existing preoperative assessment tools, including ASA status and Lee and Eagle scores, it improved the predictive power of each tool. ${ }^{50}$ In another prospective study of 189 patients who were undergoing major general, oncologic, and urologic procedures, Revenig et $\mathrm{al}^{51}$ reported that $22.8 \%$ of patients were intermediately frail and 3.7\% were frail per the Hopkins Frailty Score. On multivariate analysis, only a patient's composite frailty score was predictive of a postoperative complication, while higher hemoglobin was protective against adverse postoperative outcomes. $^{51}$

While these frailty assessment tools are comprehensive, they involve performance-based measurements that may be impractical in preoperative clinic setting. There have been attempts to use simpler methods to assess preoperative frailty. By comparing the original Canadian Study of Health and Aging Frailty Index with variables measured in the National Surgical Quality Improvement Program (NSQIP), the modified Frailty Index (mFI) was developed. It includes eleven items that can be assessed without hands-on measurements (Table 2). ${ }^{52}$ Farhat et $\mathrm{al}^{52}$ reported that, using the $\mathrm{mFI}$ in the NSQIP database of 35,334 patients 60 years of age and older undergoing emergency general surgery, increasing $\mathrm{mFI}$ was associated with an increase in wound infection, wound occurrence, any infection, any occurrence of complications, and mortality. In multivariate logistic regression analyses comparing the effect of wound class, ASA class, age, and $\mathrm{mFI}$, the $\mathrm{mFI}$ was the strongest predictor of death among all variables. ${ }^{51}$ In another study using the NSQIP database of 6,727 inpatients who underwent operations performed by surgeons specializing in otolaryngology, Adams et $\mathrm{al}^{53}$ reported that frailty, as measured with $\mathrm{mFI}$, was associated with serious complications and higher mortality.

Table 2 Eleven items of the modified Frailty Index

History of diabetes mellitus
History of congestive heart failure
History of hypertension requiring medication
History of either transient ischemic attack or cerebrovascular accident
Functional status 2 (not independent)
History of myocardial infarction
History of either peripheral vascular disease or rest pain
History of cerebrovascular accident with neurological deficit
History of either COPD or pneumonia
History of either prior PCI, PCS, or angina
History of impaired sensorium
Notes: Functional status measured in the 30 days prior to surgery. The presence
of each variable was scored as I point. The score ranges 0 -II, with a score 0
representing absence of frailty, while a score of II represents highest degree of
frailty.
Abbreviations: COPD, chronic obstructive pulmonary disease; PCI, percutaneous
coronary intervention; PCS, prior cardiac surgery.


Frailty is a common condition among elderly patients, and it is a significant predictor of postoperative morbidity and mortality. Frailty assessment tools that are both performanceand nonperformance-based predict postoperative outcomes. The choice among multiple frailty measurement tools can be individualized to the preoperative practice environment, physicians, and staff.

\section{Nutritional status}

Increasing age is associated with an unhealthier nutritional status due to decreased access to wholesome food, reduced appetite, dental diseases, chronic diseases, medications, changes in metabolism, and psychological issues. Kaiser et a ${ }^{54}$ reported that the prevalence of malnutrition among the elderly was $22.8 \%$, with considerable differences between the settings studied (rehabilitation, 50.5\%; hospital, 38.7\%; nursing home, $13.8 \%$; community, $5.8 \%$ ). Multiple scoring systems have been developed to assess patients' nutritional status. Traditional scoring systems are based on mathematical equations using objective measures of nutritional status (eg, Nutritional Risk Index [NRI], Table 3). ${ }^{55}$ Although these nutrition scoring systems are reliable and objective tools to identify patients who are malnourished, their ability to detect patients who are "at risk" of malnutrition or to identify those who are mildly malnourished is limited. To overcome this limitation, the subjective global assessment (SGA) of nutritional status tool was developed (Table 3). SGA provides a comprehensive appraisal of nutritional status, based on clinical judgment of a few subscales. ${ }^{56}$ The MNA-sf is another nonmathematical assessment tool that consists of anthropometric measures and a questionnaire on food intake, weight loss, mobility, psychological stress/acute illness, and neuropsychological problems (Table 3). ${ }^{57,58}$ The European Society for Clinical Nutrition and Metabolism advocates the use of the MNA as a screening tool, while members of the American Society for Parenteral and Enteral Nutrition (ASPEN) do not recommend any one screening tool over another. ${ }^{59}$ In a prospective study of 100 patients undergoing major abdominal surgery, Sungurtekin et $\mathrm{al}^{60}$ reported that $44 \%$ of the patients were malnourished according to the SGA, while $61 \%$ of the patients were malnourished according to the NRI. The prevalence of malnutrition measured by these two tools increased to $67 \%$ and $82 \%$, respectively, at the time of discharge. Higher mortality was reported for the malnourished group, while no mortality was reported in the well-nourished group. The odds ratios for risk of complications were 4.4 and 9.9 in the malnourished group compared to the well-nourished group by SGA and NRI, respectively. In another study of 460 patients (mean age 55.3 years) who were undergoing major elective surgery, Kuzu et a ${ }^{61}$ reported the frequency of malnutrition as $58 \%, 64 \%$, and $67 \%$ per SGA, NRI, and the Maastricht Index (Table 3). ${ }^{62}$ Morbidity, particularly severe infection, was significantly higher among malnourished patients, with the odds ratio for morbidity ranging from 2.30 to 3.47 in the well-nourished vs malnourished patients. ${ }^{61}$

\section{Serum albumin and prealbumin}

There is some controversy surrounding the utility of albumin as marker of nutritional status. However, regardless of its

Table 3 Nutritional risk scoring models

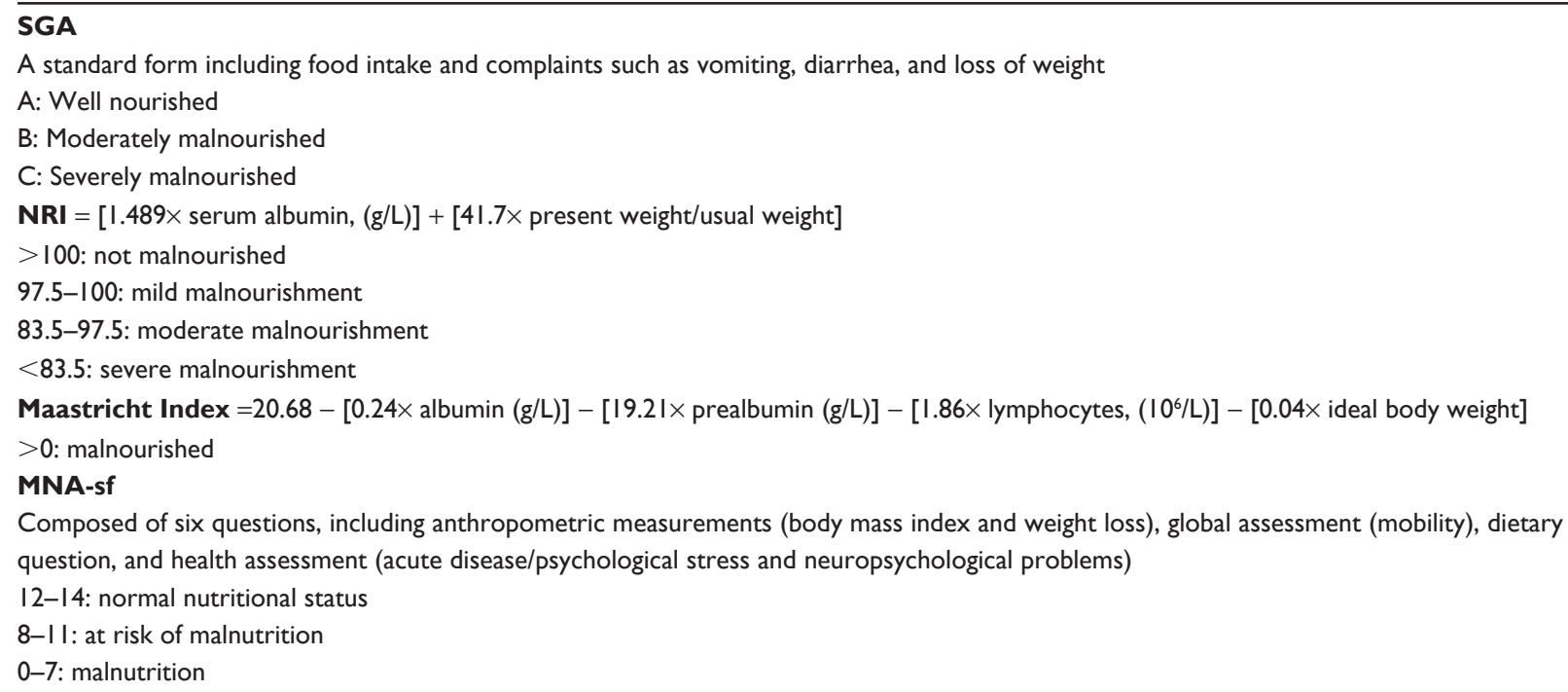


effectiveness as a nutritional marker, preoperative albumin levels have been shown to predict postoperative outcomes. In a study of 87,078 major noncardiac operations at 44 Veterans Affairs Medical Centers, Khuri et a ${ }^{63}$ assessed perioperative risk using 67 variables. They found 44 variables to be significant in predicting 30-day postoperative mortality; of these, serum albumin was the most important predictor of 30-day mortality. In another study of 81,719 cases at 44 Veterans Affairs Medical Centers, Arozullah et $\mathrm{al}^{64}$ reported the incidence of postoperative respiratory failure - defined as mechanical ventilation for $>48$ hours after surgery or reintubation and mechanical ventilation after postoperative extubation - as 3.4\%. They reported age, low albumin, and low functional status as risk factors for postoperative respiratory failure, along with abdominal aortic aneurysm repair, thoracic surgery, neurosurgery, upper abdominal surgery, peripheral vascular surgery, neck surgery, emergency surgery, high blood urea nitrogen level, and chronic obstructive pulmonary disease (COPD). Interestingly, in a systematic review of 15 articles on nutritional status and postoperative complications, van Stijn et a $1^{59}$ reported that only weight loss of $\geq 10 \%$ in the previous 6 months and low serum albumin were significant predictors of postoperative outcomes after general surgery.

Prealbumin, another hepatic protein known to reflect nutritional status, has some benefits over albumin. First, it has a shorter half-life (2 days) when compared to albumin (20 days), ${ }^{65}$ making it a more ideal indicator of acute changes in nutritional status. Second, because prealbumin is not affected by hydration status, it may be a more reliable marker of nourishment than albumin, particularly in those patients who have greater fluid fluctuations (eg, dialysis patients, congestive heart failure). ${ }^{66}$ Also, multiple studies have shown the usefulness of prealbumin in the perioperative setting. In a prospective cohort study of 114 patients who were undergoing cytoreduction surgery for ovarian cancer, Geisler et a ${ }^{67}$ reported that low prealbumin $(<10 \mathrm{mg} / \mathrm{dL})$ was associated with increased complication and mortality. In a retrospective study of 641 consecutive patients who underwent surgeries for gastrointestinal malignancy, Lin et al ${ }^{65}$ reported that hypoprealbuminemia $(<18 \mathrm{mg} / \mathrm{dL})$ predicted overall morbidity and infectious complications. In a study of 69 patients undergoing cardiac surgery, low prealbumin level $(<20 \mathrm{mg} / \mathrm{dL})$ was a risk factor for postoperative infection and need for longer mechanical ventilation. ${ }^{68}$

Since hypoalbuminemia and hypoprealbuminemia are predictors of poor postoperative outcomes, there have been attempts to improve outcomes by improving preoperative nutritional status. In a clinical trial of total parenteral nutrition (TPN) in 395 malnourished patients who were undergoing laparotomy or noncardiac thoracotomy, no differences in rates of major complications or mortality were observed. Interestingly, the investigators found more infectious complications in the TPN group. ${ }^{69}$ In another study of 167 patients who were scheduled for gastrectomy or pancreaticoduodenectomy for malignancy, Klek et $\mathrm{al}^{70}$ enrolled patients with severe nutritional deficiency, defined as one or more of the following: 1) weight loss of $10 \%$ to $15 \%$ over 5 months; 2) BMI $<18 \mathrm{~kg} / \mathrm{m}^{2}$; 3) SGA grade C; or 4) serum albumin $<3 \mathrm{~g} / \mathrm{dL}$ (without evidence of hepatic or renal dysfunction). All patients received TPN for 14 days preoperatively, and then were randomized to receive 1) standard enteral nutrition, 2) immunomodulating enteral nutrition, 3) standard parenteral nutrition, or 4) immunomodulating parenteral nutrition. Baseline serum albumin levels at study entry were 3.0-3.1 g/dL, and there were no differences between groups. After 14 days of TPN, albumin levels remained low, suggesting that albumin is a poor marker of nutritional status and adequacy of nutritional support. Postoperative nutrition therapy was continued for at least 7 days. The incidence of complications did not differ between groups. This study highlights the interesting point that hypoalbuminemia in the perioperative setting could not be corrected with rather extensive nutrition support with TPN, enteral nutrition, or immunomodulatory therapy. ${ }^{70}$

\section{Functional status}

Although not routinely included in the preoperative assessment, functional status is known to be a significant predictor of postoperative outcomes. Functional status can be measured using multiple tools, with a questionnaire being the simplest and the most commonly used. In a study of 586 patients who were undergoing major nonemergent, noncardiac surgery, Legner et al $^{71}$ assessed patients' self-reported exercise tolerance and explored the relationship with unanticipated nursing home placement. Fifty-five percent of the patients in the study reported poor preoperative physical exercise tolerance, defined as the inability to walk four blocks and climb two flights of stairs without symptomatic limitation. Overall, $9 \%$ of the patients had unanticipated nursing home placement postoperatively. Following multivariate analyses, poor exercise tolerance was a significant predictor of unanticipated nursing home placement, along with increasing age, unmarried status, living alone, five or more medications, anesthesia of $>4$ hours, orthopedic surgery, and serious postoperative complications. Arozullah et $\mathrm{al}^{64}$ developed a postoperative 
pneumonia index system using the NSQIP database. These investigators identified that the type of surgery (abdominal aortic aneurysm repair, thoracic, upper abdominal, neck, neurosurgery, and vascular surgery), increasing age, poor functional status, weight loss of $>10 \%$ in the last 6 months, history of COPD, general anesthesia, impaired sensorium, history of cerebrovascular accident, blood urea nitrogen level, amount of transfusion, emergency surgery, chronic steroid use, current smoker (within 1 year), and alcohol intake of two drinks per day in the past 2 weeks were risk factors associated with postoperative pneumonia.

Also, functional dependence has been reported as a risk factor for postoperative surgical site infection. In a prospective case-control study of 150 patients with methicillin-resistant Staphylococcus aureus surgical site infection (MRSA SSI) compared to 231 age-matched, uninfected control patients and 128 control patients with SSI due to methicillin-susceptible S. aureus (MSSA SSI), Chen et al ${ }^{72}$ measured ASA physical classification score, burden of comorbidity (Charlson score), National Nosocomial Infections Surveillance risk index score (NNIS), and ADLs. They reported that more patients in the MRSA SSI group compared to the uninfected control group required assistance with bathing $(32 \%$ vs $11 \% ; P<0.001)$, dressing (32\% vs $11 \%$; $P<0.001)$, feeding (12\% vs 4\%; $P=0.003)$, and walking $(47 \%$ vs $26 \%, P<0.001)$. The same trend was observed when the MRSA SSI group was compared to the MSSA SSI group, with more assistance required for dressing (32\% vs $10.3 \%$; $P<0.001)$, feeding (12\% vs $11 \% ; P<0.001)$, and walking ( $47 \%$ vs $27 \% ; P=0.001$ ). In the multivariate analysis, the need for assistance with three or more ADLs, Medicaid as the sole form of health care coverage, a wound classification of $>2$, duration of surgery longer than the NNIS 75th percentile for surgical duration, and obesity were significant independent predictors of MRSA SSI. When the MRSA SSI group was compared with the MSSA SSI group, need for assistance with three or more ADLs, duration of surgery longer than the NNIS 75th percentile for surgical duration, and congestive heart failure were significant predictors of MRSA SSI. The same investigators conducted a retrospective nested case-control study involving seven hospitals, using a similar design. They identified 86 patients who developed MRSA SSI, 167 patients who underwent surgery and did not develop SSI, and 64 patients who developed MSSA SSI. They found that patients with MRSA SSI had poorer functional status than controls and more often lacked independence in ADLs, including ambulation ( $45 \%$ vs $28 \%, P=0.009)$, bathing $(37 \%$ vs $15 \% ; P<0.001)$, dressing $(37 \%$ vs $15 \% ; P<0.001)$, and feeding $(15 \%$ vs $6 \% ; P=0.02)$. In the multivariate analysis, lack of independence in ADLs, Charlson score, and wound class were independent predictors of MRSA SSI. When the MRSA SSI group was compared with the MSSA SSI group, the MRSA SSI group more frequently lacked independence with ambulation (45\% vs $23 \% ; P=0.009)$, bathing $(37 \%$ vs $13 \% ; P=0.003)$, dressing $(37 \%$ vs $13 \% ; P=0.003)$, and feeding $(15 \%$ vs $2 \% ; P=0.03)$. In the multivariate analysis, lack of independence with ADLs, Charlson score, wound class, and surgical duration were independent predictors of MRSA SSI. ${ }^{72}$

Functional status can be assessed easily even in a busy preoperative setting. Poor functional status has been reported to be associated with nursing home placement, postoperative pulmonary complications, and MRSA SSI. Assessment of functional status can provide valuable information with regard to predicting outcomes; an issue that is important not only to surgeons but also to patients and their families.

\section{Cognitive assessment}

The prevalence of dementia and cognitive impairment without dementia in those aged 71 years and older in the United States in 2002 was reported as $13.9 \%$ and $22.3 \%$, respectively. ${ }^{73,74}$ Dementia is a known risk factor for morbidity and mortality in the geriatric population. ${ }^{73}$ Postoperative delirium is a common postoperative complication with reported incidence of $32 \%-42 \%{ }^{75-77}$ Multiple studies show the strong relationship between preoperative cognitive impairment and postoperative delirium.

In a prospective cohort study, Galanakis et al ${ }^{78}$ looked at 105 consecutive patients who underwent hip surgery for hip fracture repair or elective hip replacement. Using the Mini Mental State Exam (MMSE), they reported a 23.8\% incidence of a postoperative acute confusional status among older patients (mean age 74.9 years). In the multivariate analysis, higher age, prior cognitive impairment, depression, low educational level, and preoperative abnormal sodium were independent predictors of postoperative delirium. MMSE is a well-validated method to assess cognitive function. However, its 30 questions may be cumbersome to use in a busy PAC. ${ }^{79}$ Marcantonio et $\mathrm{al}^{80}$ reported similar results in another prospective study of 1,341 patients who were undergoing general, orthopedic, and gynecologic surgery. They assessed patients' cognitive function using the Telephone Interview for Cognitive Status, which is a modification of the MMSE that avoids the need for written responses. These investigators also assessed the patients' physical function using the Specific Activity Scale, a questionnaire for self-administered 
functional classification that is ordinally scaled from I (best) to IV (worst). The postoperative occurrence rate of delirium was $9 \%$. Following multivariate analysis, age $\geq 70$ years, alcohol abuse, low Telephone Interview for Cognitive Status score (cognitive impairment), Specific Activity Scale class IV (severe physical impairment), markedly abnormal preoperative sodium, potassium, or glucose level, aortic aneurysm surgery, and noncardiac thoracic surgery independently predicted delirium. The occurrence of postoperative delirium in this study was also associated with major postoperative complications, death, LOS, and discharge to long-term care or rehabilitative facilities.

Preoperative cognitive impairment also predicts postoperative pulmonary complications. In a prospective cohort study of 48 patients $>70$ years of age who were undergoing elective coronary artery bypass graft surgery, Aykut et al ${ }^{81}$ assessed patients' cognitive function using the Montreal Cognitive Assessment test (MoCA). ${ }^{82}$ MoCA is a 30-point test that is well validated in the setting of mild cognitive impairment. Patients were classified as mildly cognitively impaired (MoCA score of 19-25) or not impaired (MoCA score $>25$ ). Patients with mild cognitive impairment had significant deterioration in their spirometry performance, higher atelectasis, and longer need for mechanical ventilation. ${ }^{81}$

Studies also exist that show the relationship between preoperative cognitive impairment and postoperative morbidity. Robinson et $\mathrm{al}^{83}$ found that baseline cognitive impairment associated with an increase in one or more postoperative complications, independent of age, comorbidities, and ASA classification among 186 patients aged 65 years and older who were undergoing an elective operation requiring postoperative intensive care unit (ICU) admission. While multiple methods are available to assess a patient's cognitive abilities preoperatively, ${ }^{79,82,84}$ the recent ACS/AGS guideline endorses the use of the Mini-Cog Test. ${ }^{85}$ The Mini-Cog allows clinicians to quickly assess numerous cognitive domains including cognitive function, memory, language comprehension, visual-motor skills, and executive function. The incidence of impaired cognitive function in surgical patients between the ages of 60 years and 90 years was reported to be $44 \%$, with a range of $17 \%-100 \%$, using the Mini-Cog. ${ }^{83}$

\section{Mood/depression}

An estimated 7 million adults $\geq 65$ years of age, ie, $15 \%-20 \%$ of adults, are affected by depression. ${ }^{86}$ The prevalence of depression among elderly patients in the preoperative period is even higher. In a prospective cohort study of 149 hip and 133 knee arthroplasty patients, the prevalence of anxiety and depression, as measured by the Hospital Anxiety Outcome Score was $27.9 \%$ (hip), $20.3 \%$ (knee), and 33.6\% (hip) and $22.7 \%$ (knee), respectively, which drops after surgery. ${ }^{87}$ Duivenvoorden et al ${ }^{87}$ further found that patients with preoperative anxiety and depressive symptoms had worse patientreported outcomes. In another study using the 2008 National Inpatient Sample of 292,191 patients undergoing colon and/ or rectal surgery, the prevalence of depression, identified by International Classification of Diseases- 9 codes, was $6.9 \%{ }^{88}$ Patients with depression had longer LOSs and a higher likelihood of skilled nursing requirement upon discharge. After surgery for thoracic neoplasm, patients who were $\geq 70$ years of age and who screened positive for depression by the Geriatric Depression Scale were at increased risk for having major complications and being discharged to a non-home location. ${ }^{89}$ In a study of 817 patients who were undergoing coronary artery bypass graft surgery, Blumenthal et a $1^{90}$ used the selfreported Center for Epidemiological Studies-Depression measure to assess mental state. The prevalence of mild depression was $26 \%$, while moderate-to-severe depression was $12 \%$. In the survival analysis, after controlling for age, sex, number of grafts, diabetes, smoking, left ventricular ejection fraction, and previous myocardial infarction, patients with baseline severe depression and mild or moderate-to-severe depression that persisted from baseline to 6 months had a higher mortality rate..$^{90}$ Using the Mental Health Inventory depression screen, Ho et a ${ }^{91}$ reported $29.2 \%$ of 648 patients undergoing valve surgery were depressed at baseline. Depression was a significant predictor of mortality at 6 months, along with COPD, hypertension, age, and serum creatinine.

Depression is common among older patients in the perioperative period. The prevalence of depression is higher when proactively screened compared to detecting it from prior history. Depression is reported to be associated with patient-reported outcomes, major complications, discharge to a place other than the patient's home, complications, and mortality.

\section{Comprehensive geriatric assessment}

As reviewed previously, physiologic changes with age involve multiple organ systems, and, thus, accurate measurement of physiologic changes might involve more than one set of measurements. Several studies have used comprehensive geriatric assessments to explore their association with postoperative outcomes, as summarized in Table 4 . Indeed, the physiologic changes that combat surgical stressors also involve multiple organ systems, with correlations 


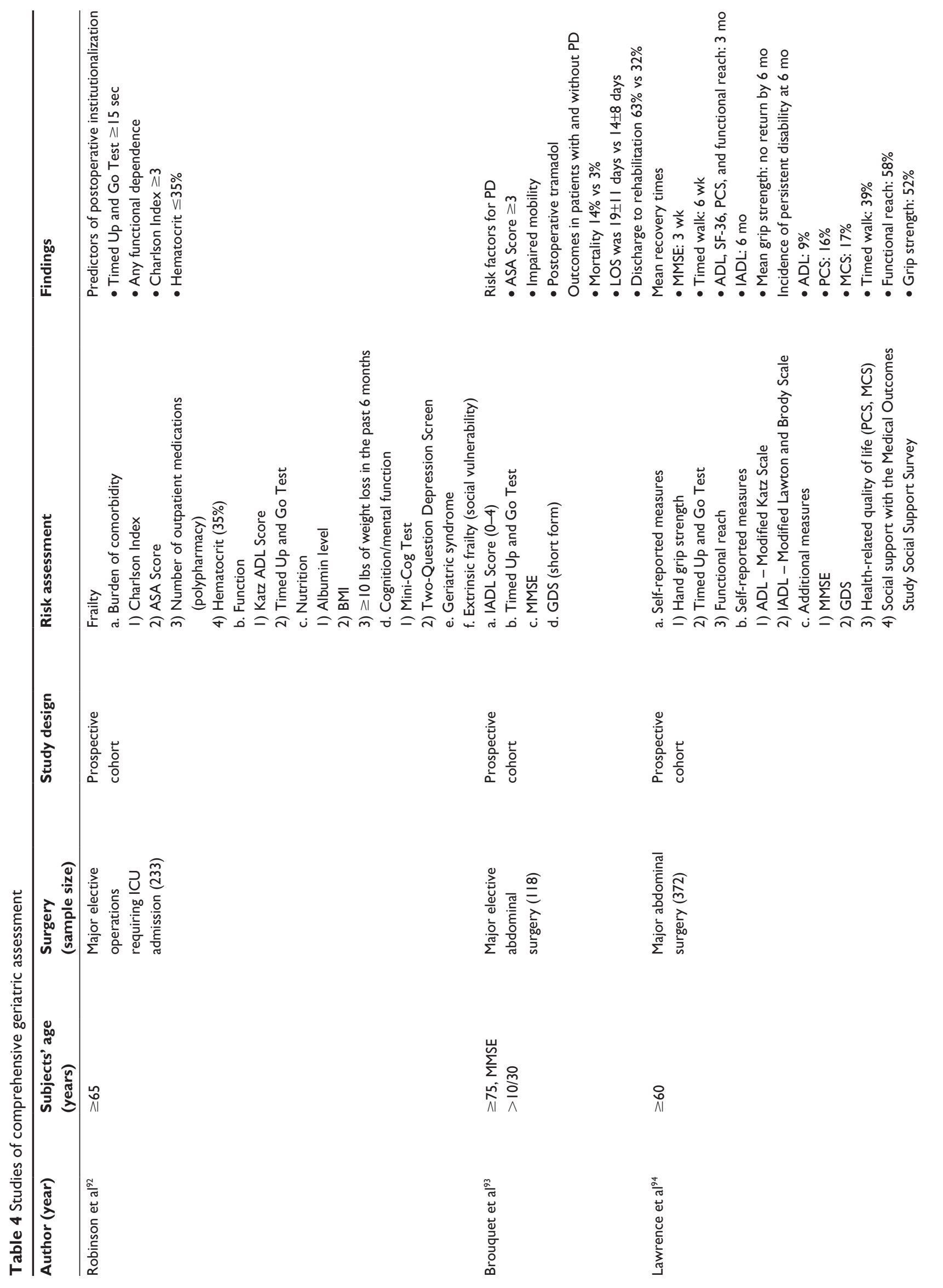



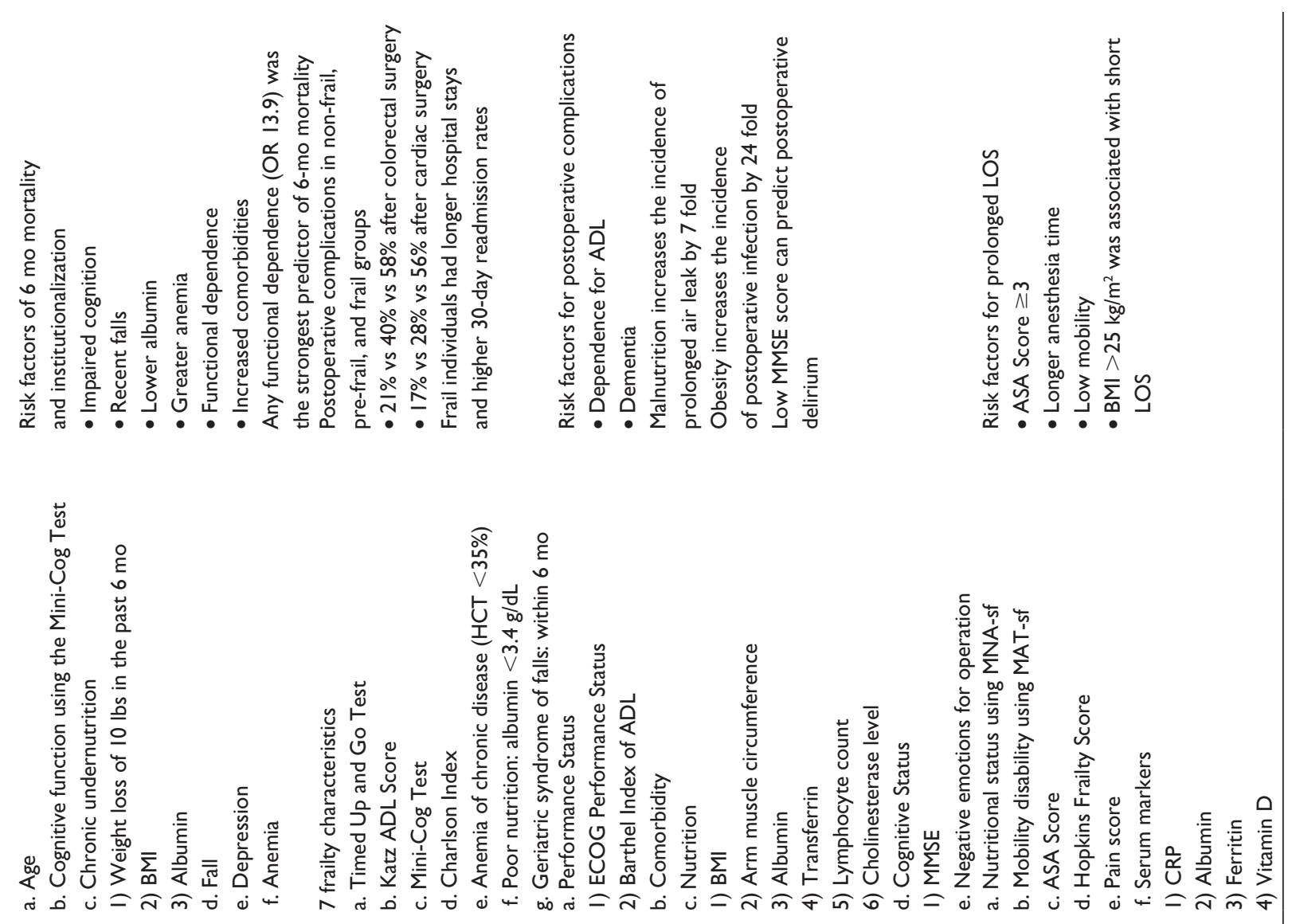

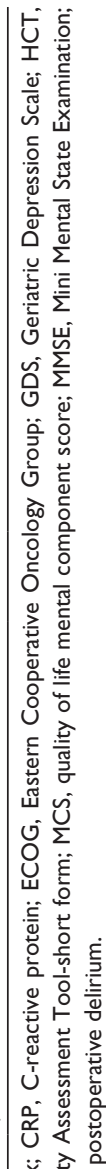

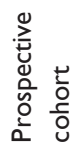
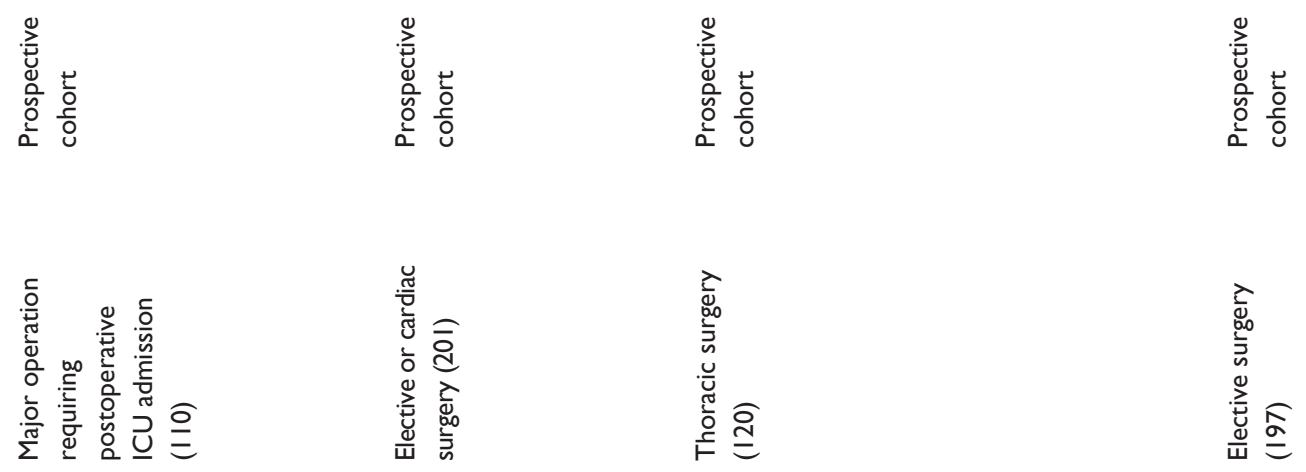

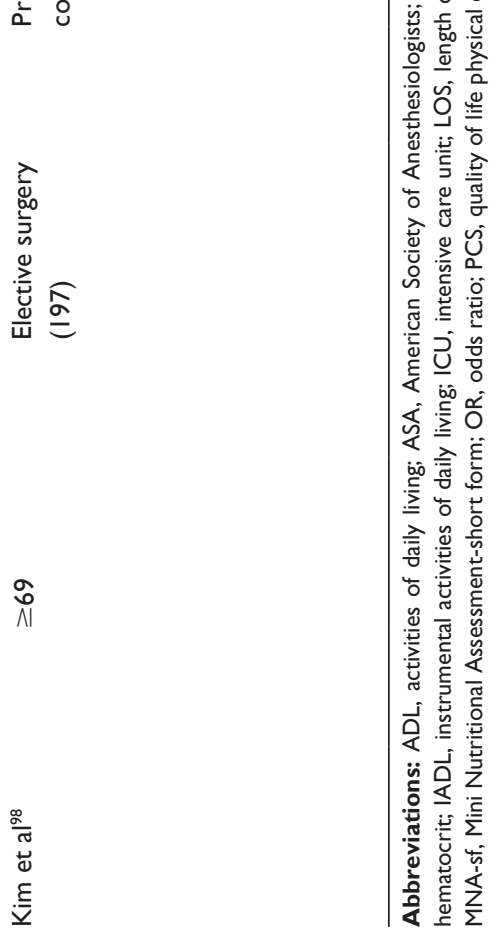

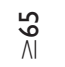

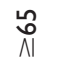

읏

aิ

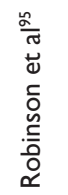

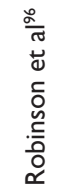

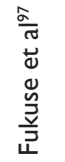

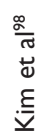


present between the various systems. Preoperative geriatric assessments require multiple domains, and inclusion of multiple geriatric-related domains may therefore better predict postoperative outcomes. Further research is needed to help identify the most comprehensive and efficient geriatric assessment tools that can be used to identify "risky" older patients for elective surgery so that appropriate and realistic perioperative care plans can be established.

\section{Anesthetic techniques: is there a safer way to anesthetize the elderly?}

The field of anesthesiology has seen major improvement in safety over the past 3 decades; anesthesia mortality rates have decreased from two deaths per 10,000 anesthetics administered in the 1980 s to about one death per 200,000-300,000 anesthetics administered today. As our population continues to age, anesthesiologists are tasked with identifying anesthetic techniques that minimize morbidity and mortality and postoperative cognitive disorders in the older patient.

Multiple studies have demonstrated an association between neuraxial (epidural or spinal) anesthesia and improved perioperative outcomes, especially in the orthopedic patient population. In perhaps the most expansive population study to date in this area, Memtsoudis et al ${ }^{99}$ conducted a retrospective cohort study looking at 795,135 patient records (mean age $=65.7$ years) who underwent total hip arthroplasty or total knee arthroplasty. Using multivariate logistic regressions, they found that neuraxial anesthesia was associated with decreased odds for combined major complications (OR ranged from 0.87 to 0.89 depending on the age group, $P<0.001$ ), need for intensive care services (OR ranged from 0.56 to 0.63 depending on the age group, $P<0.001$ ), and prolonged LOS (OR ranged from 0.63 to 0.78 depending on the age group, $P<0.001)$ compared with general anesthesia in all patient subgroups. For patients without major cardiopulmonary comorbidities, the added benefit of neuraxial anesthesia increased with increasing age.

Detecting specific anesthetic techniques aimed at reducing postoperative cognitive disorders has the potential to considerably improve the perioperative care delivered to elderly surgical patients. Postoperative cognitive disorders describe a wide spectrum of neurocognitive or postoperative dysfunction (impairment in cognitive function) to dementia (impairment in memory) to postoperative delirium (impairment in consciousness). ${ }^{100}$ In a randomized, prospective cohort of 2,000 elderly patients (mean age $70.1 \pm 4$.6 years) undergoing general anesthesia and major noncardiac surgery, Cai et $\mathrm{al}^{101}$ found significantly decreased MMSE scores days 1-3 after surgery in the group receiving isoflurane, an inhalational anesthetic, in comparison to the total intravenous anesthesia (TIVA) group. It is well established that TIVA is associated with a lesser activation of the stress response, as evidenced by serum cortisol, lower plasma levels of NE, epinephrine, and growth hormone, and lesser heart rate variability. However, it is important to note several limitations of this study: anesthetic depth was not clearly defined in the study for either group; and a relatively higher than normal concentration of isoflurane ( $2 \%-3 \%$ end-tidal concentration) was used for maintenance of anesthesia, which may partially explain the decrease in postoperative MMSE scores in the inhalational group.

Anesthetic technique is a potentially adjustable risk factor for postoperative delirium. Unfortunately, most studies have been unable to detect a consistent difference in postoperative delirium between regional and general anesthesia. ${ }^{102}$ This inconsistency may be partially clarified by the sedation levels that often accompany regional or neuraxial anesthesia. Propofol is an intravenous anesthetic that is commonly used as an adjuvant sedative during regional or neuraxial anesthesia. Seiber et al conducted a double-blinded, randomized controlled trial of 114 patients 65 years or older undergoing hip fracture repair with spinal anesthesia and propofol sedation. ${ }^{103}$ Depth of propofol sedation was monitored via the Bispectral index (BIS). The authors found that the use of "light" sedation (BIS of 80) decreased the prevalence of postoperative delirium by $50 \%$ compared with the "deep" sedation group (BIS less than or equal to 50).

Anesthesia choice and management is important to consider in elderly surgical patients given their vulnerability to stress and the disproportionate risk of costly perioperative complications. Studies involving regional versus general anesthesia have demonstrated a significant impact on perioperative outcomes such as LOS and ICU utilization in patients receiving regional anesthesia; however, a causal relationship between regional anesthesia and improved postoperative cognitive dysfunction remains elusive. Robust studies about other anesthesia techniques such as TIVA versus general anesthesia are lacking, with the exception of one study performed by Cai et $\mathrm{al}^{101}$ which has considerable limitations.

\section{Summary}

The aging population will significantly increase the demand for surgical services, and perioperative care for the elderly may be different than for others. Accordingly, preoperative evaluation should be different in younger and older patients, particularly since currently used perioperative risk assessment tools (eg, RCRI) focus on one system, and most older patients exhibit declining physiologic function and reserves in multiple systems. Specifically, impaired ADL and IADL due to walk- 
ing and balance issues are common in older and frail persons, and are associated with loss of independence, disability, and death. Physical and mental functional impairments, from acute illness or surgical stress, impair quality of life, compromise independence, limit future therapeutic options, and increase mortality. Mobility is a functional component of frailty that requires the coordinated effort of sensory, neural, and musculoskeletal systems. Cognitive abilities, including those used in planning and monitoring performance (eg, executive function) also have a key role in mobility. Risk factors for common cardiac and vascular diseases are linked to both declining mobility and cognition. As such, it is our conviction that quick and easy-to-perform mobility testing (eg, Get Up and Go Test; 5-m walk speed; Mobility Assessment Tool-short form) may better predict postoperative status of elderly patients after a surgical procedure than traditional risk assessment tools focused on single organ systems or even multidomain frailty assessment tools. Future prospective studies are needed that focus on time-efficient screening methodologies of both physiologic and cognitive reserves in older patients scheduled for surgery within a busy preoperative clinic setting, so that at-risk, vulnerable elders can be identified early and proactive perioperative management strategies (eg, strength, balance, and mobility prehabilitation) enabled to safeguard against morbidity and reassure maintenance of independence in this large segment of American population.

\section{Acknowledgment}

This was funded in part by a grant from the Anesthesia Patient Safety Foundation (LG), National Institutes of Health/ National Institutes on Aging grants AG-042758, AG-033727 (LG), and pilot funds from the Wake Forest Translational Science Center and Center of Integrative Medicine (LG) at Wake Forest School of Medicine, Winston Salem, NC.

We also acknowledge our patient volunteers who participated in our institutional review board-approved geriatric preoperative assessment study (IRB number 00019392).

\section{Disclosure}

The authors report not conflicts of interest in this work.

\section{References}

1. Schoufour JD, Mitnitski A, Rockwood K, Evenhuis HM, Echteld MA. Development of a frailty index for older people with intellectual disabilities: results from the HA-ID study. Res Dev Disabil. 2013;34(5):1541-1555.

2. Hermans H, Evenhuis HM. Multimorbidity in older adults with intellectual disabilities. Res Dev Disabil. 2014;35(4):776-783.

3. de Kuijper G, Mulder H, Evenhuis H, Scholte F, Visser F, Hoekstra PJ. Determinants of physical health parameters in individuals with intellectual disability who use long-term antipsychotics. Res Dev Disabil. 2013;34(9):2799-2809.
4. Goldman L, Caldera DL, Nussbaum SR, et al. Multifactorial index of cardiac risk in noncardiac surgical procedures. NEnglJMed. 1977;297(16): 845-850.

5. Gupta PK, Franck C, Miller WJ, Gupta H, Forse RA. Development and validation of a bariatric surgery morbidity risk calculator using the prospective, multicenter NSQIP dataset. J Am Coll Surg. 2011;212(3): 301-309.

6. Lee TH, Marcantonio ER, Mangione CM, et al. Derivation and prospective validation of a simple index for prediction of cardiac risk of major noncardiac surgery. Circulation. 1999;100(10):1043-1049.

7. Fleisher LA, Beckman JA, Brown KA, et al. ACC/AHA 2007 guidelines on perioperative cardiovascular evaluation and care for noncardiac surgery: executive summary: a report of the American College of Cardiology/ American Heart Association Task Force on Practice Guidelines (Writing Committee to Revise. Anesth Analg. 2008;106(3):685-712.

8. Qaseem T. Risk assessment for and strategies to reduce perioperative pulmonary complications. Ann Intern Med. 2006;145(7):553. Author reply 553 .

9. Oresanya LB, Lyons WL, Finlayson E. Preoperative assessment of the older patient: a narrative review. JAMA. 2014;311(20):2110-2120.

10. Vincent GK, Velkoff VA. The next four decades, the older population in the United States: 2010-2050. Current Population Reports. US Census Bureau, Washington, DC; 2010:P25-1138. Available from: www.census.gov/prod/2010pubs/p25-1138.pdf. October 8, 2013.

11. Hall MJ, DeFrances CJ, Williams SN, Golosinskiy A, Schwartzman A. National Hospital Discharge Survey: 2007 summary. Natl Health Stat Report. 2010;29:1-20, 24.

12. Etzioni DA, Liu JH, O’Connell JB, Maggard MA, Ko CY. Elderly patients in surgical workloads: a population-based analysis. Am Surg. 2003;69(11):961-965.

13. Turrentine FE, Wang H, Simpson VB, Jones RS. Surgical risk factors, morbidity, and mortality in elderly patients. J Am Coll Surg. 2006;203(6):865-877.

14. Wanebo HJ, Cole B, Chung M, et al. Is surgical management compromised in elderly patients with breast cancer? Ann Surg. 1997;225(5): 579-586.

15. Kehlet H, Nielsen HJ. Impact of laparoscopic surgery on stress responses, immunofunction, and risk of infectious complications. New Horiz. 1998; 6(2 suppl):S80-S88.

16. Wilmore DW. From Cuthbertson to fast-track surgery: 70 years of progress in reducing stress in surgical patients. Ann Surg. 2002;236(5):643-648.

17. Kehlet H. Multimodal approach to control postoperative pathophysiology and rehabilitation. Br J Anaesth. 1997;78(5):606-617.

18. Burton D. Endocrine and metabolic response to surgery. Contin Educ Anaesth Crit Care Pain. 2004;4(5):144-147.

19. Lakatta EG, Levy D. Arterial and cardiac aging: major shareholders in cardiovascular disease enterprises: part I: aging arteries: a "set up" for vascular disease. Circulation. 2003;107(1):139-146.

20. Lakatta EG, Levy D. Arterial and cardiac aging: major shareholders in cardiovascular disease enterprises: part II: the aging heart in health: links to heart disease. Circulation. 2003;107(2):346-354.

21. Seals DR, Monahan KD, Bell C, Tanaka H, Jones PP. The aging cardiovascular system: changes in autonomic function at rest and in response to exercise. Int J Sport Nutr Exerc Metab. 2001;11(suppl):S189-S195.

22. Monahan KD. Effect of aging on baroreflex function in humans. Am J Physiol Regul Integr Comp Physiol. 2007;293(1):R3-R12.

23. Desborough JP. The stress response to trauma and surgery. Br J Anaesth. 2000;85(1):109-117.

24. Mulholland MW, Lillemoe KD, Doherty GM, Maier RV, Simeone DM, Upchurch GR. Greenfield's Surgery: Scientific Principles and Practice. 5th ed. Philadelphia: Wolters Kluwer Health/Lippincott Williams \& Wilkins; 2012.

25. Watters JM, Clancey SM, Moulton SB, Briere KM, Zhu JM. Impaired recovery of strength in older patients after major abdominal surgery. Ann Surg. 1993;218(3):380-390. 
26. Lunardi AC, Miranda CS, Silva KM, Cecconello I, Carvalho CRF. Weakness of expiratory muscles and pulmonary complications in malnourished patients undergoing upper abdominal surgery. Respirology. 2012;17(1):108-113.

27. Kimura F, Shimizu H, Yoshidome H, Ohtsuka M, Miyazaki M. Immunosuppression following surgical and traumatic injury. Surg Today. 2010;40(9):793-808.

28. Ogawa K, Hirai M, Katsube T, et al. Suppression of cellular immunity by surgical stress. Surgery. 2000;127(3):329-336.

29. Jameson P, Desborough JP, Bryant AE, Hall GM. The effect of cortisol suppression on interleukin- 6 and white blood cell responses to surgery. Acta Anaesthesiol Scand. 1997;41(2):304-308.

30. Lin E, Calvano SE, Lowry SF. Inflammatory cytokines and cell response in surgery. Surgery. 2000;127(2):117-126.

31. Decker D, Schondorf M, Bidlingmaier F, Hirner A, von Ruecker AA. Surgical stress induces a shift in the type-1/type-2 T-helper cell balance, suggesting down-regulation of cell-mediated and up-regulation of antibody-mediated immunity commensurate to the trauma. Surgery. 1996;119(3):316-325.

32. Rymkiewicz PD, Heng YX, Vasudev A, Larbi A. The immune system in the aging human. Immunol Res. 2012;53(1-3):235-250.

33. Singh $T$, Newman AB. Inflammatory markers in population studies of aging. Ageing Res Rev. 2011;10(3):319-329.

34. Chung HY, Cesari M, Anton S, et al. Molecular inflammation: underpinnings of aging and age-related diseases. Ageing Res Rev. 2009; 8(1):18-30.

35. Krabbe KS, Pedersen M, Bruunsgaard H. Inflammatory mediators in the elderly. Exp Gerontol. 2004;39(5):687-699.

36. Bruunsgaard H, Pedersen M, Pedersen BK. Aging and proinflammatory cytokines. Curr Opin Hematol. 2001;8(3):131-136.

37. Jess P, Schultz K, Bendtzen K, Nielsen OH. Systemic inflammatory responses during laparoscopic and open inguinal hernia repair: a randomised prospective study. Eur J Surg. 2000;166(7):540-544.

38. Karayiannakis AJ, Makri GG, Mantzioka A, Karousos D, Karatzas G. Systemic stress response after laparoscopic or open cholecystectomy: a randomized trial. Br J Surg. 1997;84(4):467-471.

39. Huang T-J, Hsu RW-W, Li Y-Y, Cheng C-C. Less systemic cytokine response in patients following microendoscopic versus open lumbar discectomy. J Orthop Res. 2005;23(2):406-411.

40. Marana E, Colicci S, Meo F, Marana R, Proietti R. Neuroendocrine stress response in gynecological laparoscopy: TIVA with propofol versus sevoflurane anesthesia. J Clin Anesth. 2010;22(4):250-255.

41. Ledowski T, Bein B, Hanss R, et al. Neuroendocrine stress response and heart rate variability: a comparison of total intravenous versus balanced anesthesia. Anesth Analg. 2005;101(6):1700-1705.

42. Xue Q-L. The frailty syndrome: definition and natural history. Clin Geriatr Med. 2011;27(1):1-15.

43. Fried LP, Xue QL, Cappola AR, et al. Nonlinear multisystem physiological dysregulation associated with frailty in older women: implications for etiology and treatment. J Gerontol A Biol Sci Med Sci. 2009;64(10):1049-1057.

44. Boyd CM, Xue Q-L, Simpson CF, Guralnik JM, Fried LP. Frailty, hospitalization, and progression of disability in a cohort of disabled older women. Am J Med. 2005;118(11):1225-1231.

45. Fried LP, Tangen CM, Walston J, et al; Cardiovascular Health Study Collaborative Research Group. Frailty in older adults: evidence for a phenotype. J Gerontol A Biol Sci Med Sci. 2001;56(3): M146-M156.

46. Rockwood K, Song X, MacKnight C, et al. A global clinical measure of fitness and frailty in elderly people. CMAJ. 2005;173(5):489-495.

47. Jones D, Song X, Mitnitski A, Rockwood K. Evaluation of a frailty index based on a comprehensive geriatric assessment in a population based study of elderly Canadians. Aging Clin Exp Res. 2005; 17(6):465-471.

48. Rolfson DB, Majumdar SR, Tsuyuki RT, Tahir A, Rockwood K. Validity and reliability of the Edmonton Frail Scale. Age Ageing. 2006; 35(5):526-529.
49. Dasgupta M, Rolfson DB, Stolee P, Borrie MJ, Speechley M. Frailty is associated with postoperative complications in older adults with medical problems. Arch Gerontol Geriatr. 2009;48(1):78-83.

50. Makary MA, Segev DL, Pronovost PJ, et al. Frailty as a predictor of surgical outcomes in older patients. J Am Coll Surg. 2010;210(6): 901-908.

51. Revenig LM, Canter DJ, Taylor MD, et al. Too frail for surgery? Initial results of a large multidisciplinary prospective study examining preoperative variables predictive of poor surgical outcomes. $\mathrm{J} \mathrm{Am} \mathrm{Coll}$ Surg. 2013;217(4):665.e1-670.e1.

52. Farhat JS, Velanovich V, Falvo AJ, et al. Are the frail destined to fail? Frailty index as predictor of surgical morbidity and mortality in the elderly. J Trauma Acute Care Surg. 2012;72(6):1526-1530; discussion $1530-1531$.

53. Adams P, Ghanem T, Stachler R, Hall F, Velanovich V, Rubinfeld I. Frailty as a predictor of morbidity and mortality in inpatient head and neck surgery. JAMA Otolaryngol Head Neck Surg. 2013;139(8):783-789.

54. Kaiser MJ, Bauer JM, Rämsch C, et al; Mini Nutritional Assessment International Group. Frequency of malnutrition in older adults: a multinational perspective using the mini nutritional assessment. J Am Geriatr Soc. 2010;58(9):1734-1738.

55. Wolinsky FD, Coe RM, McIntosh WA, et al. Progress in the development of a nutritional risk index. J Nutr. 1990;120(suppl):1549-1553.

56. Detsky AS, McLaughlin JR, Baker JP, et al. What is subjective global assessment of nutritional status? JPEN J Parenter Enteral Nutr. 1987; 11(1):8-13.

57. Cohendy R, Rubenstein LZ, Eledjam JJ. The Mini Nutritional Assessment-Short Form for preoperative nutritional evaluation of elderly patients. Aging (Milano). 2001;13(4):293-297.

58. Kaiser MJ, Bauer JM, Ramsch C, et al; MNA-International Group. Validation of the Mini Nutritional Assessment short-form (MNA-SF): a practical tool for identification of nutritional status. $J$ Nutr Health Aging. 2009;13(9):782-788.

59. van Stijn MFM, Korkic-Halilovic I, Bakker MSM, van der Ploeg T, van Leeuwen PAM, Houdijk APJ. Preoperative nutrition status and postoperative outcome in elderly general surgery patients: a systematic review. JPEN J Parenter Enteral Nutr. 2013;37(1):37-43.

60. Sungurtekin H, Sungurtekin U, Balci C, Zencir M, Erdem E. The influence of nutritional status on complications after major intraabdominal surgery. J Am Coll Nutr. 2004;23(3):227-232.

61. Kuzu MA, Terzioğlu H, Genç V, et al. Preoperative nutritional risk assessment in predicting postoperative outcome in patients undergoing major surgery. World J Surg. 2006;30(3):378-390.

62. De Jong PC, Wesdorp RI, Volovics A, Roufflart M, Greep JM, Soeters PB. The value of objective measurements to select patients who are malnourished. Clin Nutr. 1985;4(2):61-66.

63. Khuri SF, Daley J, Henderson W, et al. Risk adjustment of the postoperative mortality rate for the comparative assessment of the quality of surgical care: results of the National Veterans Affairs Surgical Risk Study. J Am Coll Surg. 1997;185(4):315-327.

64. Arozullah AM, Daley J, Henderson WG, Khuri SF. Multifactorial risk index for predicting postoperative respiratory failure in men after major noncardiac surgery. The National Veterans Administration Surgical Quality Improvement Program. Ann Surg. 2000;232(2):242-253.

65. Lin MY, Liu WY, Tolan AM, Aboulian A, Petrie BA, Stabile BE. Preoperative serum albumin but not prealbumin is an excellent predictor of postoperative complications and mortality in patients with gastrointestinal cancer. Am Surg. 2011;77(10):1286-1289.

66. Mears E. Outcomes of continuous process improvement of a nutritional care program incorporating serum prealbumin measurements. Nutrition. 1996;12(7-8):479-484.

67. Geisler JP, Linnemeier GC, Thomas AJ, Manahan KJ. Nutritional assessment using prealbumin as an objective criterion to determine whom should not undergo primary radical cytoreductive surgery for ovarian cancer. Gynecol Oncol. 2007;106(1):128-131.

68. Yu PJ, Cassiere HA, Dellis SL, Manetta F, Kohn N, Hartman AR. Impact of preoperative prealbumin on outcomes after cardiac surgery. JPEN JParenter Enteral Nutr. 2014;pii:0148607114536735. [Epub ahead of print]. 
69. Perioperative total parenteral nutrition in surgical patients. The Veterans Affairs Total Parenteral Nutrition Cooperative Study Group. N Engl J Med. 1991;325(8):525-532.

70. Klek S, Sierzega M, Szybinski P, et al. Perioperative nutrition in malnourished surgical cancer patients - a prospective, randomized, controlled clinical trial. Clin Nutr. 2011;30(6):708-713.

71. Legner VG, Doerner D, Reilly DF, McCormick WC. Risk factors for nursing home placement following major nonemergent surgery. Am J Med. 2004;117(2):82-86.

72. Chen T-Y, Anderson DJ, Chopra T, Choi Y, Schmader KE, Kaye KS. Poor functional status is an independent predictor of surgical site infections due to methicillin-resistant Staphylococcus aureus in older adults. J Am Geriatr Soc. 2010;58(3):527-532.

73. Plassman BL, Langa KM, Fisher GG, et al. Prevalence of cognitive impairment without dementia in the United States. Ann Intern Med. 2008;148(6):427-434.

74. Plassman BL, Langa KM, Fisher GG, et al. Prevalence of dementia in the United States: the aging, demographics, and memory study. Neuroepidemiology. 2007;29(1-2):125-132.

75. Van der Mast RC, Roest FH. Delirium after cardiac surgery: a critical review. J Psychosom Res. 1996;41(1):13-30.

76. Dyer CB, Ashton CM, Teasdale TA. Postoperative delirium. A review of 80 primary data-collection studies. Arch Intern Med. 1995;155(5):461-465.

77. Sasajima Y, Sasajima T, Uchida H, et al. Postoperative delirium in patients with chronic lower limb ischaemia: what are the specific markers? Eur J Vasc Endovasc Surg. 2000;20(2):132-137.

78. Galanakis P, Bickel H, Gradinger R, Von Gumppenberg S, Förstl H. Acute confusional state in the elderly following hip surgery: incidence, risk factors and complications. Int J Geriatr Psychiatry. 2001;16(4): 349-355.

79. Folstein MF, Folstein SE, McHugh PR. "Mini-mental state". A practical method for grading the cognitive state of patients for the clinician. J Psychiatr Res. 1975;12(3):189-198.

80. Marcantonio ER, Goldman L, Mangione CM, et al. A clinical prediction rule for delirium after elective noncardiac surgery. JAMA. 1994;271(2):134-139.

81. Aykut K, Albayrak G, Guzeloglu M, Baysak A, Hazan E. Preoperative mild cognitive dysfunction predicts pulmonary complications after coronary artery bypass graft surgery. J Cardiothorac Vasc Anesth. 2013 27(6):1267-1270.

82. Nasreddine ZS, Phillips NA, Bédirian V, et al. The Montreal Cognitive Assessment, MoCA: a brief screening tool for mild cognitive impairment. J Am Geriatr Soc. 2005;53(4):695-699.

83. Robinson TN, Wu DS, Pointer LF, Dunn CL, Moss M. Preoperative cognitive dysfunction is related to adverse postoperative outcomes in the elderly. J Am Coll Surg. 2012;215(1):12-17.

84. Borson S, Scanlan J, Brush M, Vitaliano P, Dokmak A. The mini-cog: a cognitive "vital signs" measure for dementia screening in multi-lingual elderly. Int J Geriatr Psychiatry. 2000;15(11):1021-1027.

85. Chow WB, Rosenthal RA, Merkow RP, Ko CY, Esnaola NF. Optimal preoperative assessment of the geriatric surgical patient: a best practices guideline from the American College of Surgeons National Surgical Quality Improvement Program and the American Geriatrics Society. J Am Coll Surg. 2012;215(4):453-466.

86. Aldrich N. CDC promotes public health approaches to address depression among older adults. Available from: www.cdc.gov/aging/pdf/ cib_mental_health.pdf

Clinical Interventions in Aging

\section{Publish your work in this journal}

Clinical Interventions in Aging is an international, peer-reviewed journal focusing on evidence-based reports on the value or lack thereof of treatments intended to prevent or delay the onset of maladaptive correlates of aging in human beings. This journal is indexed on PubMed Central, MedLine,
87. Duivenvoorden T, Vissers MM, Verhaar JA, et al. Anxiety and depressive symptoms before and after total hip and knee arthroplasty: a prospective multicentre study. Osteoarthritis Cartilage. 2013;21(12): 1834-1840.

88. Balentine CJ, Hermosillo-Rodriguez J, Robinson CN, Berger DH, Naik AD. Depression is associated with prolonged and complicated recovery following colorectal surgery. J Gastrointest Surg. 2011; 15(10):1712-1717.

89. Yesavage JA, Brink TL, Rose TL, et al. Development and validation of a geriatric depression screening scale: a preliminary report. JPsychiatr Res. 1982-1983;17(1):37-49.

90. Blumenthal JA, Lett HS, Babyak MA, et al; NORG Investigators. Depression as a risk factor for mortality after coronary artery bypass surgery. Lancet. 2003;362(9384):604-609.

91. Ho PM, Masoudi FA, Spertus JA, et al. Depression predicts mortality following cardiac valve surgery. Ann Thorac Surg. 2005;79(4): 1255-1259.

92. Robinson TN, Wallace JI, Wu DS, et al. Accumulated frailty characteristics predict postoperative discharge institutionalization in the geriatric patient. J Am Coll Surg. 2011;213(1):37-42; discussion 42-44.

93. Brouquet A, Cudennec T, Benoist S, et al. Impaired mobility, ASA status and administration of tramadol are risk factors for postoperative delirium in patients aged 75 years or more after major abdominal surgery. Ann Surg. 2010;251(4):759-765.

94. Lawrence VA, Hazuda HP, Cornell JE, et al. Functional independence after major abdominal surgery in the elderly. J Am Coll Surg. 2004;199(5):762-772.

95. Robinson TN, Eiseman B, Wallace JI, et al. Redefining geriatric preoperative assessment using frailty, disability and co-morbidity. Ann Surg. 2009;250(3):449-455.

96. Robinson TN, Wu DS, Pointer L, Dunn CL, Cleveland JC, Moss M. Simple frailty score predicts postoperative complications across surgical specialties. Am J Surg. 2013;206(4):544-550.

97. Fukuse T, Satoda N, Hijiya K, Fujinaga T. Importance of a comprehensive geriatric assessment in prediction of complications following thoracic surgery in elderly patients. Chest. 2005;127(3):886-891.

98. Kim S, Edwards A, Rustowicz L, et al. Do sex differences in preoperative mobility, nutrition and frailty status predict length of hospital stay among older noncardiac surgical patients? J Am Geriatr Soc. 2013;61(s1):B155.

99. Memtsoudis SG, Rasul R, Suzuki S, et al. Does the impact of the type of anesthesia on outcomes differ by patient age and comorbidity burden? Reg Anesth Pain Med. 2014;39(2):112-119.

100. Wu CL, Hsu W, Richman JM, Raja SN. Postoperative cognitive function as an outcome of regional anesthesia and analgesia. Reg Anesth Pain Med. 2012;29(3):257-268.

101. Cai Y, Hu H, Liu P, et al. Association between the apolipoprotein E4 and postoperative cognitive dysfunction in elderly patients undergoing intravenous anesthesia and inhalation anesthesia. Anesthesiology 2012;116(1):84-93.

102. Bryson GL, Wyand A. Evidence-based clinical update: general anesthesia and the risk of delirium and postoperative cognitive dysfunction. Can J Anaesth. 2006;53(7):669-677.

103. Sieber FE, Zakriya KJ, Gottschalk A, et al. Sedation depth during spinal anesthesia and the development of postoperative delirium in elderly patients undergoing hip fracture repair. Mayo Clin Proc. 2010;85(1):18-26.

\section{Dovepress}

CAS, Scopus and the Elsevier Bibliographic databases. The manuscript management system is completely online and includes a very quick and fair peer-review system, which is all easy to use. Visit http://www.dovepress. com/testimonials.php to read real quotes from published authors. 